\title{
Canny Edge Detection Algorithm Application for Analysis of the Potential Field Map
}

\author{
A. Eshaghzadeh and R.S Kalantari \\ Institute of Geophysics, University of Tehran, Iran \\ E-mail: eshaghzadeh.ata@gmail.com
}

\begin{abstract}
Determination of potential fields' anomaly borders is a useful help to their interpretation. There is various technique of edge detecting that is applied in image processing. In this paper, the canny edge detection (CED) method has been proposed as boundary enhancement of the magnetic and gravity potential field data. The Canny operator works in a multi-stage process. This method is based on the characteristic of intensity values of considered pixel. The edge detector should have a good signal-to-noise ratio, so that edges can be found even if potential field data quality is poor. For 2-dimensional bounds, residual potential field map is first smoothed by using a 2-D Gaussian filter. Afterwards, computing the horizontal gradients of the smoothed map and then using the gradient magnitude and direction to estimate borders strength and direction at every pixel. The Canny edge detection algorithm uses double threshold for edges revelation. In this research, a new procedure to define the thresholds has been suggested. The results obtained from the synthetic data set, with and without random noise, have been discussed. The method is demonstrated on real gravity and magnetic data set surveyed from Iran. The CED results are compared with three common methods as edge detector, namely the analytic signal, tilt angle and total horizontal derivative of the tilt angle.
\end{abstract}

Keywords: canny edge detection (CED), Gaussian filter, horizontal gradients, potential fields

\section{INTRODUCTION}

Earth potential fields' anomalies are as a result of the geological sources interposition with different shapes, depths and of course density characteristics and susceptibility of gravity and magnetic fields respectively. Edge detection algorithms are employed to analyze potential field's data sets, such as gravity and magnetic maps, in order to detect main geological bodies, subtle geological features, geological structures and alignments (Boschetti, 2005). There are many techniques such as downward continuation, horizontal and vertical derivatives, local phase filters and etc. that can be employed to detect the edges and borders of geological structures. Edge detecting of a potential field map reduces the amount of data significantly and consequently useless information is filtered out, while preserving the important features properties in a map. An approach to the conventional phase filter by Miller and Singh (1994) introduced, named "Tilt angle filter" that is ratio of the value of vertical derivative to the amplitude of total horizontal derivative of potential field data. Wijns et al. (2005) introduced the Theta map which is based upon the analytic 
signal. Verduzco et al. (2004) recommended using the total horizontal derivative of the tilt angle (which they termed the THDR) as an edge detector. Pilkington and Keating (2004) show that the THDR is closely related to the local (instantaneous) frequency as defined by Thurston and Smith (1997). Cooper and Cowan (2008) suggested using a filter based on the ratio of related normalized standard deviations (NSTD) to make large and small-amplitude edges visible simultaneously. Eshaghzadeh (2014) used of the normalized standard deviations for microgravity data analysis. Li et al. (2013) indicated the enhanced mathematical morphology filter as edge detection in potential-field data. Eshaghzadeh (2015) demonstrated the acceptable performance the tilt angle of the total horizontal gradient normalized by the analytic signal as an image edge detector of the gravity field. Eshaghzadeh (2016) proposed a new local phase filter namely the tilt angle of the balanced total horizontal derivative for gravity anomalies edge intensification. Pal and Majumdar (2015) applied the first vertical derivative, second vertical derivative, analytical signal and tilt angle or derivative for geological appraisal over the Singhbhum-Orissa craton, India using GOCE, EIGEN6-C2 and in situ gravity data. Narayan et al. (2016) delineated structural features over a part of the Bay of Bengal using total and balanced horizontal derivative techniques.

The Canny edge detection algorithm is known to many as the optimal edge detector. Alike local phase filters, canny procedure is also based on smoothed potential data gradients. In this study, Canny method (1986) is applied for analysis and interpretation of the gravity and magnetic data of Iran. The results show high performance and efficiency of this technique on the potential field map.

\section{METHODOLOGY}

The first step of implementing the CED algorithm is to apply the 2-D Gaussian filter to smooth the residual anomaly map of the potential field before trying to locate and detect any edges. Because the Gaussian filter can be computed using a simple mask, it is used particularly in the Canny algorithm. Assume $f(x, y)$ is the potential field data, thus:

$$
\mathrm{z}(\mathrm{x}, \mathrm{y} ; \sigma)=g(x, y ; \sigma) * f(x, y)=\sum_{k=0}^{x-1} \sum_{l=0}^{y-1} \mathrm{~g}(k, l ; \sigma) f(x-k, y-l)
$$

Where $z(x, y, \sigma)$ is the smoothed data and $\sigma$ is the standard deviation of the Gaussian filter, which controls the degree of smoothing, given by:

$$
g_{x, y, \sigma}=\frac{1}{\sqrt{2 \pi \sigma^{2}}} \exp \left(-\frac{x^{2}+y^{2}}{2 \sigma^{2}}\right)
$$

The size of the filter (S) for a given Gaussian standard deviation $(\sigma)$ is chosen based on:

$$
S=2 \times \operatorname{ceil}(2 \times \sigma)+1
$$

The "ceil" operator rounds the elements between the brackets to the nearest integers greater than or equal to it. Table-1 shows the relation between the sigma and the Gaussian filter kernel size (convolution mask) as for equation 3. A convolution mask is much smaller than the actual image. It is important to select the suitable size for the Gaussian kernel that will affect the performance of the CED. The larger size of the window, the lower detector's sensitivity to noise. 
Besides, the edge positioning and detection error will slightly increase with the increase of the Gaussian filter kernel size. The sigma value shouldn't be smaller than 0.5 .

Table-1: Relation between the sigma and the Gaussian filter kernel size

$\begin{array}{cc}\text { Sigma }(\sigma) & \text { Mask size }(\mathrm{S}) \\ 0.5 & 3 \times 3 \\ 1 & 5 \times 5 \\ 2 & 9 \times 9 \\ 3 & 13 \times 13 \\ 4 & 19 \times 19\end{array}$

After smoothing the map and eliminating the noise, the next step is calculating the horizontal gradients. Canny (1986) showed that this filter can be well approximated by first-order derivatives of Gaussians. The gradient magnitudes (also known as the edge strengths) at each pixel is calculated as the norm of the Corresponding gradient vector, i.e.

$$
\|F\|_{x, y}=\sqrt{\left(\frac{\partial z}{\partial x}\right)^{2}+\left(\frac{\partial z}{\partial y}\right)^{2}}
$$

The direction of the gradient is computed as follows:

$$
\theta_{x, y}=\tan ^{-1}\left(\frac{\partial z}{\partial y} / \frac{\partial z}{\partial x}\right)
$$

Where $\left(\frac{\partial z}{\partial x}\right)$ and $\left(\frac{\partial z}{\partial y}\right)$ are the gradients of the smoothed data through $\mathrm{x}$ and $\mathrm{y}$ axis directions respectively.

Only the specific orientation can spot for the edge directions. There are four possible directions around each pixel. In the horizontal direction ( 0 and 180 degrees), along the positive diagonal (45 and 225 degrees), in the vertical direction (90 and 270 degrees) and along the negative diagonal (135 and 315 degrees). Therefore, any edge direction calculated will be round up to the closest angle (Fig. 1).

The next step is a process known as non-maximal suppression or edge thinning. In this phase, only those pixels are protected providing that represent a local maximum along the direction of the gradient, i.e., perpendicular to the edge tangent and the algorithm sets to zero all pixels that are not actually on the ridge top so as to obtain a thin border in the output. 


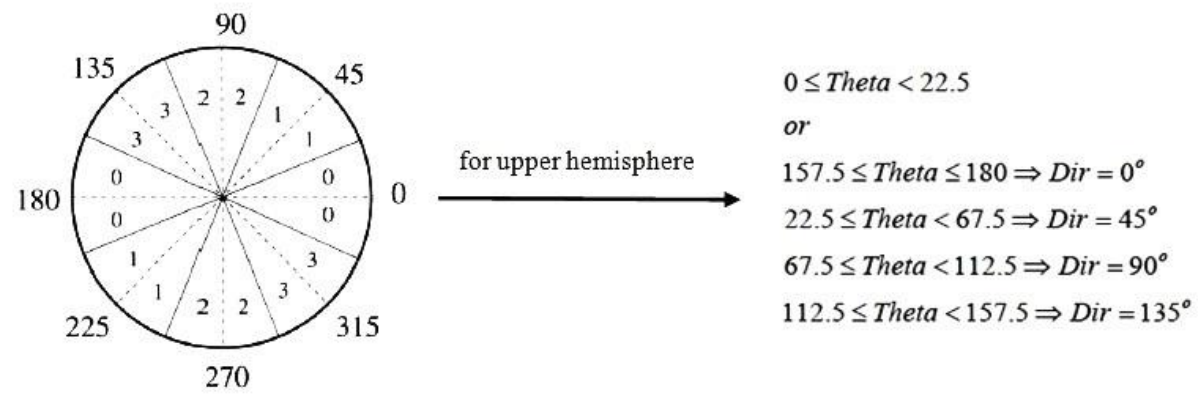

Fig. 1: The specific orientation around each pixel.

A simple example of non-maximum suppression is shown in Fig. 2. Almost all pixels have gradient directions to the upward. Therefore, they are compared to above and below pixels. The pixels that turn out to be maximal in this checking have gray background color. All other pixels will be suppressed.

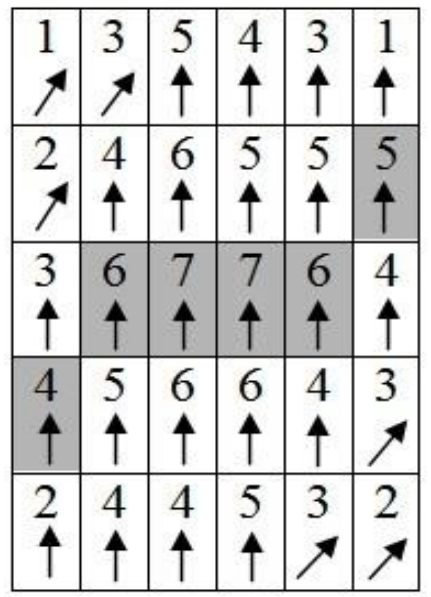

Fig. 2: Explanation of non-maximum suppression. Two upright pixel values are compared with the value in the edge direction. The resulting edge pixels have gray background color. All other pixels values are changed to zero.

In the last step, sets of linked edge pixels are concluded from the gradients magnitude values that remained unsuppressed in the previous operation. This is done with a technique called hysteresis threshold using two different threshold values $t_{h}$ (high threshold), $t_{\mathrm{l}}$ (low threshold) (with $t_{h}>t_{1}$ ) that how the threshold values selection is effective on the consequences. Each pixel in the map in which the magnitude value is greater than $t_{1}$ is supposed to be an edge pixel and pixels in which magnitude value is greater than $t_{\text {hi }}$ are accepted as edge pixels and the pixels with magnitude gradients values less than $t_{l}$ are removed eventually. The edge pixels stronger than the upper threshold are marked as strong; the edge pixels weaker than the lower threshold are suppressed and edge pixels between the two thresholds are marked as weak. Strong edges are interpreted as certain edge, and can immediately be included in the final border map. The weak edges are considered if and only if they are connected to a pixel that is greater than the high threshold, i.e. strong edges. 


\section{Threshold limit}

The threshold value in the CED algorithm is obtained by trial and error prevalently. It is tried that the optimum values for the lower and upper threshold in potential data analysis are defined. Utilizing the CED technique for several synthetic and real potential field data, it is found that the sensitivity of the CED method than the low threshold $\left(t_{1}\right)$ is very scrimp as its value can be considered as the square root of the minimum quantity of the gradient magnitudes, but slight change in the amount of high threshold is efficacious in the output. The various deliberations and examinations yielded an experiential equation for the estimation of the optimum upper threshold value (tho) which is expressed as

$t_{h o}=\sqrt{\frac{\left|s_{F}-a_{F}\right|}{m_{F}}}$

Where $s_{F}, a_{F}$ and $m_{F}$ are the standard deviation, average and maximum value of the gradient magnitudes, respectively.

\section{SYNTHETIC MODELS}

\section{Magnetic examples}

Fig.3 displays the magnetic field of the synthetic model consisting three separated rectangular blocks which have the same magnetization intensity $(10 \mathrm{~A} / \mathrm{m})$ and the declination and inclination angles for both the magnetization and geomagnetic field are assumed to be zero and 90 degrees, respectively (vertical magnetization and reduced to pole). Image size is $700 \mathrm{~m} \times$ $500 \mathrm{~m}$. In this paper we used the described method by Rao and Babu (1991) for computation of magnetic anomalies due to multiple prisms. The rectangular bodies have the same size (length $=340 \mathrm{~m}$; width $=100 \mathrm{~m}$; thickness $=100 \mathrm{~m}$ ) with their tops located at depths $10 \mathrm{~m}, 20 \mathrm{~m}$ and $30 \mathrm{~m}$, from left to right as showed in Fig. 3.

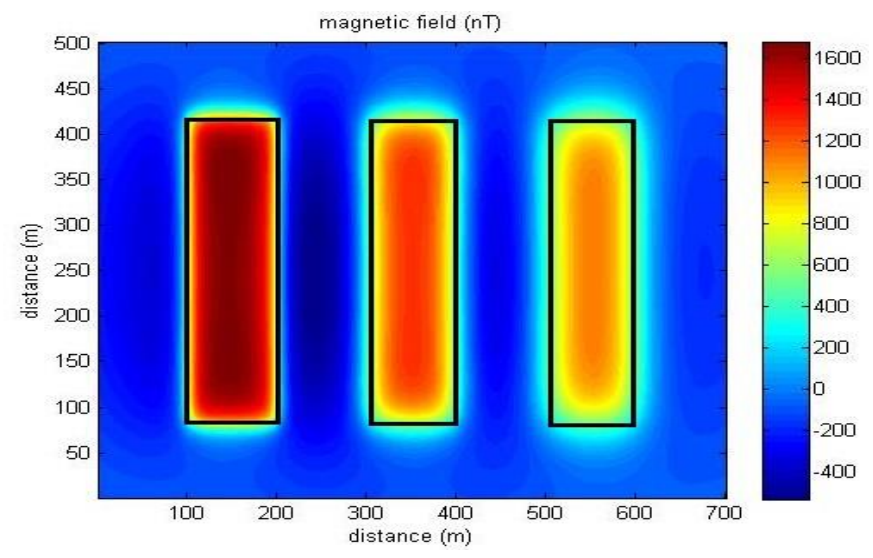

Fig.3: The magnetic field of the three separate rectangular blocks with dimensions of $340 \mathrm{~m} \times$ $100 \mathrm{~m} \times 100 \mathrm{~m}$, its outlines are shown. The magnetization intensity has been assumed $10 \mathrm{~A} / \mathrm{m}$. 
The magnetic field of the synthetic model consisting two rectangular prisms which have the same magnetization intensity $(10 \mathrm{~A} / \mathrm{m})$ into $500 \mathrm{~m} \times 500 \mathrm{~m}$ grid size. The supposed declination and inclination angles for the magnetization are zero and 90 degrees (vertical magnetization) respectively. For the geomagnetic field both angles have been assumed 90 degrees. In this model, there are two separate prisms: upper size is $340 \mathrm{~m} \times 100 \mathrm{~m} \times 20 \mathrm{~m}$ at $5 \mathrm{~m}$ depth in the north-south direction and lower size is $340 \mathrm{~m} \times 100 \mathrm{~m} \times 75 \mathrm{~m}$ at $25 \mathrm{~m}$ depth in the western-eastern direction (Left to right). Random noise with average of 1 and standard deviation of 0.5 is added to the data set. Table-2 shows the quantities of the required variables in the equation 6 and the both threshold values which were calculated for noisy and without noise data. The attained upper and lower threshold values for the synthetic model data consisting three rectangular prisms, with and without noise, are $t_{h_{0}}=0.21, t_{l}=0.068$ and $t_{h o}=0.2, t_{l}=0.07$, respectively. For synthetic model, data consisting two rectangular prisms, with and without noise, are $t_{h o}=0.254, t_{l}=0.045$ and $t_{h o}=$ $0.255, \mathrm{t}_{\mathrm{l}}=0.046$, respectively.

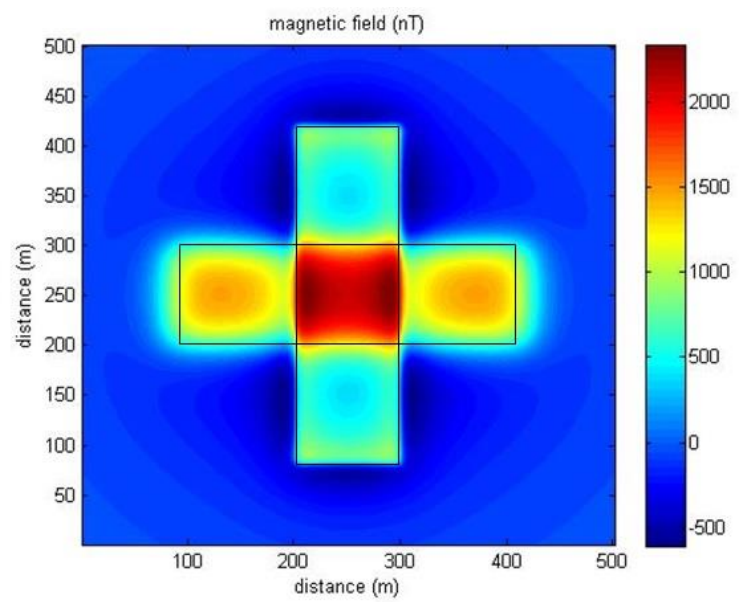

Fig. 4: The magnetic field of synthetic model consists of two rectangular prisms: the upper one is $340 \mathrm{~m} \times 100 \mathrm{~m} \times 20 \mathrm{~m}$ at $5 \mathrm{~m}$ depth in north-south direction and the lower one is $340 \mathrm{~m} \times$ $100 \mathrm{~m} \times 75 \mathrm{~m}$ at $25 \mathrm{~m}$ depth in western-eastern direction which its outlines are shown. The magnetization intensity has been assumed $10 \mathrm{~A} / \mathrm{m}$.

Table-2: The values of the required variables in the equation 6 and the upper and lower thresholds values which have been obtained from with and without noise data.

$\begin{array}{ccccccc} & \mathrm{S}_{\mathrm{F}}(\mathrm{std}) & \begin{array}{c}\mathrm{a}_{\mathrm{F}} \\ \text { (average) }\end{array} & \mathrm{m}_{\mathrm{F}}(\max ) & \min _{\mathrm{F}} & \mathrm{t}_{\mathrm{H}} & \mathrm{t}_{\mathrm{l}} \\ \begin{array}{c}3 \text { prisms model } \\ \text { without noise }\end{array} & 15.52 & 11.87 & 87.92 & 0.005 & 0.2 & 0.07 \\ \begin{array}{c}3 \text { prisms model } \\ \text { with noise }\end{array} & 15.56 & 11.82 & 88.13 & 0.0046 & 0.21 & 0.068 \\ \begin{array}{c}2 \text { prisms model } \\ \text { without noise }\end{array} & 21.34 & 11.41 & 152.7 & 0.0021 & 0.255 & 0.046 \\ \begin{array}{c}2 \text { prisms model } \\ \text { with noise }\end{array} & 21.29 & 11.43 & 152.77 & 0.002 & 0.254 & 0.045\end{array}$


The output of the CED method includes zero and one. The maximum value of the CED gives location of the body edges, as shown in Figs. 5 and 6 . The value of the Gaussian standard deviation $(\sigma)$ was selected 0.5 , i.e. the Gaussian filter kernel size is $3 \times 3$ as for equation 3 . The CED has small sensitivity to noise. Figs. 7 and 8 show the CED method results of the noisy data.

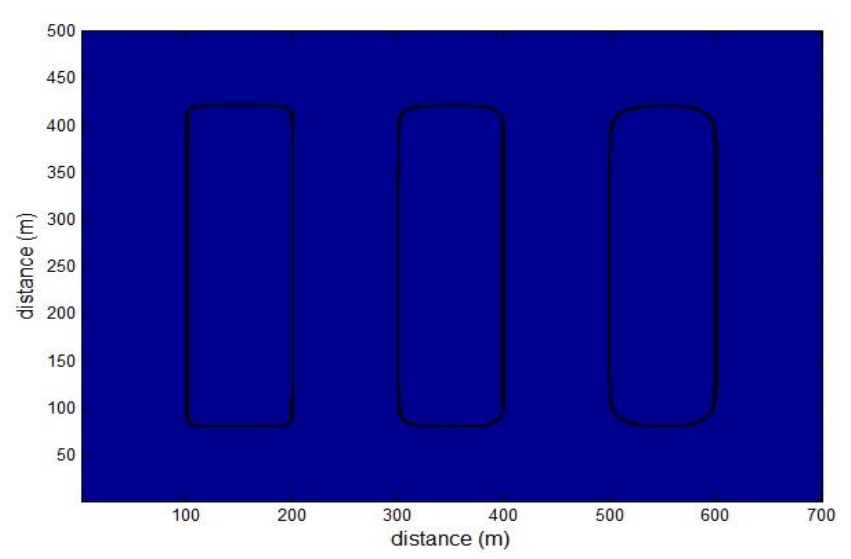

Fig. 5: The CED of the magnetic data in Fig. 3.

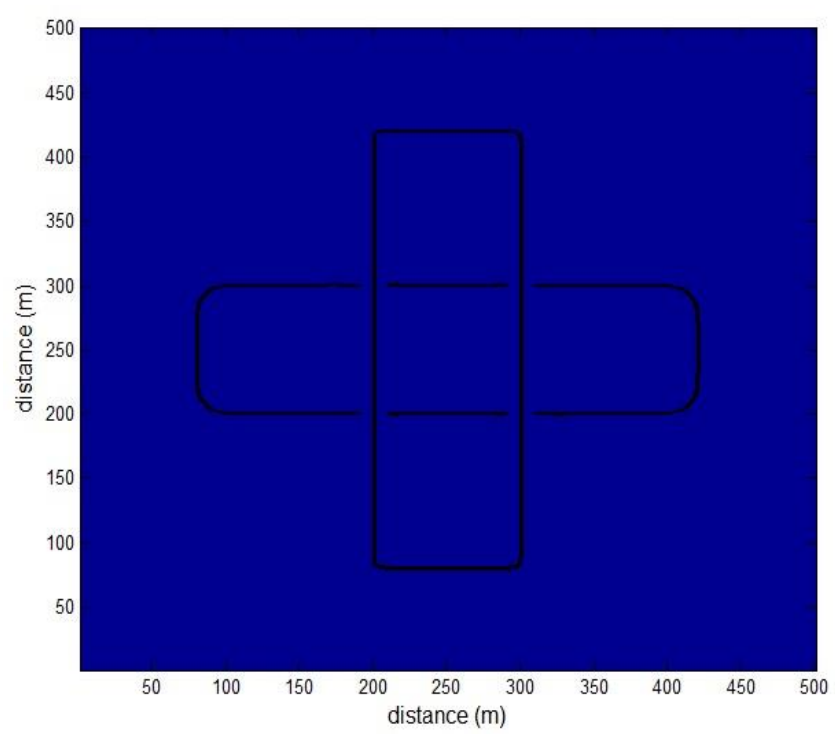

Fig. 6: The CED of the magnetic data in Fig. 4.

The CED response is almost the same as shallow and deep sources. The CED results show that in proportion to depth enhancement, the curvature of the rectangular blocks unfolded corners is increased. The accuracy of the CED algorithm outputs are accepted completely for the synthetic magnetic data in both cases, with and without noise. 


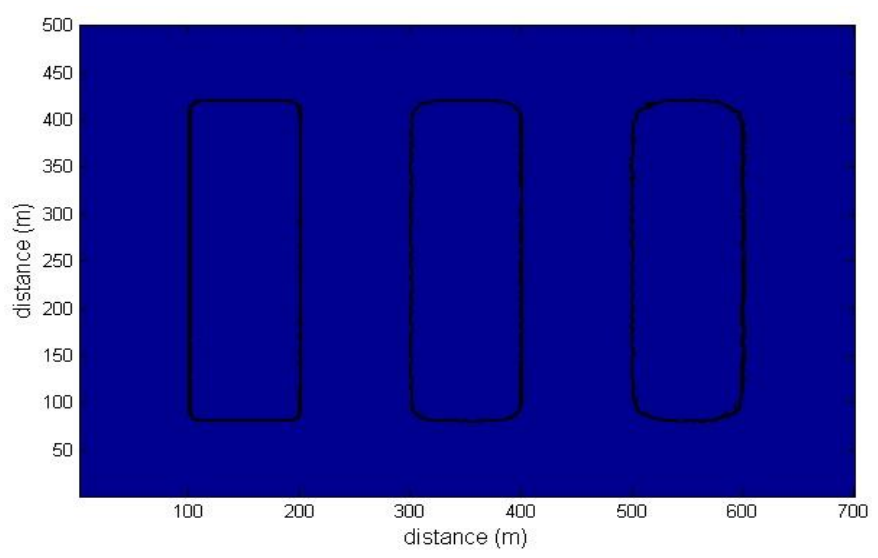

Fig. 7: The CED of the magnetic data in Fig. 3 that random noise with average and standard deviation of 1 and 0.5 respectively is added to it.

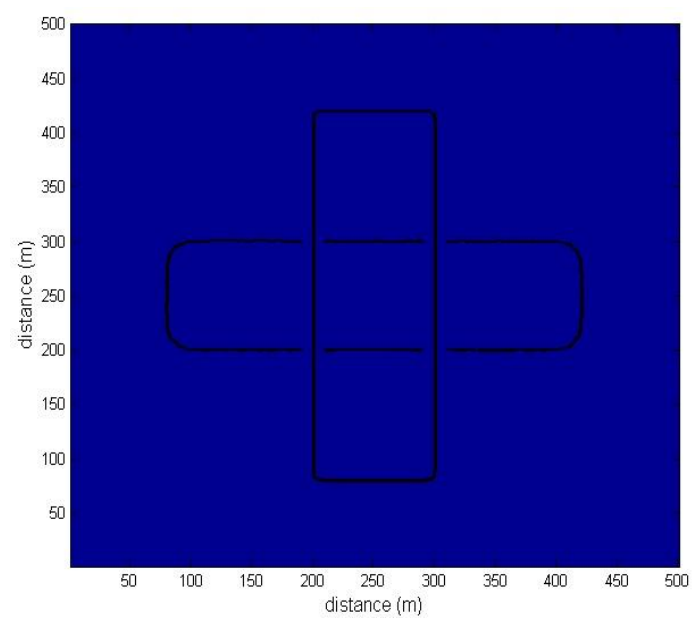

Fig. 8: The CED of the magnetic data in Fig. 4 which random noise with average and standard deviation of 1 and 0.5 respectively is added to it.

\section{Gravity example}

The total gravity field has been constructed into $400 \mathrm{~m} \times 400 \mathrm{~m}$ grid for a rectangular prism size of $300 \mathrm{~m} \times 100 \mathrm{~m} \times 40 \mathrm{~m}$ at $10 \mathrm{~m}$ depth and a sphere with the center at $110 \mathrm{~m}$ depth and 90 $\mathrm{m}$ radius, its outlines are shown in Fig. 9. The density contrast is assumed $2 \mathrm{gr} / \mathrm{cm}^{3}$ for both anomaly sources. Using the estimated gradient magnitudes from the synthetic gravity data, the threshold values are calculated. Table-3 contains the results. The achieved upper and lower threshold values for the synthetic gravity data are $t_{h o}=0.33$ and $t_{l}=0.006$ respectively. The value of the Gaussian standard deviation $(\sigma)$ is defined 0.5 .

The consequence of the CED analysis of the gravity data in the Fig. 9 is displayed in Fig.10. For the spherical source, the CED signalizes the horizontal cross section border in which the radius size is maximum. 


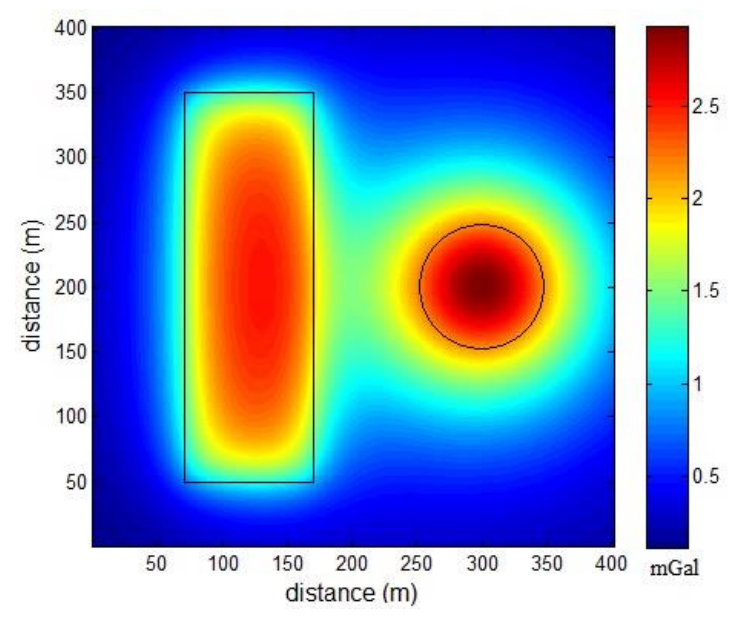

Fig. 9: The computed total gravity field for a rectangular prism size of $300 \mathrm{~m} \times 100 \mathrm{~m} \times 40 \mathrm{~m}$ at $10 \mathrm{~m}$ depth and a sphere with the center at $110 \mathrm{~m}$ depth and $90 \mathrm{~m}$ radius, its outlines are shown.

Table-3: The values of the required variables in the equation 6 and the upper and lower thresholds values which have been obtained from the synthetic gravity data.
$\mathrm{S}_{\mathrm{F}}(\mathrm{std})$
$\mathrm{a}_{\mathrm{F}}$
(average)
0.0094
$\mathrm{m}_{\mathrm{F}}(\max )$
$\min _{\mathrm{F}}$
tho
$t_{1}$
0.0144
$0.0419 \quad 0.00004$
0.33
0.006

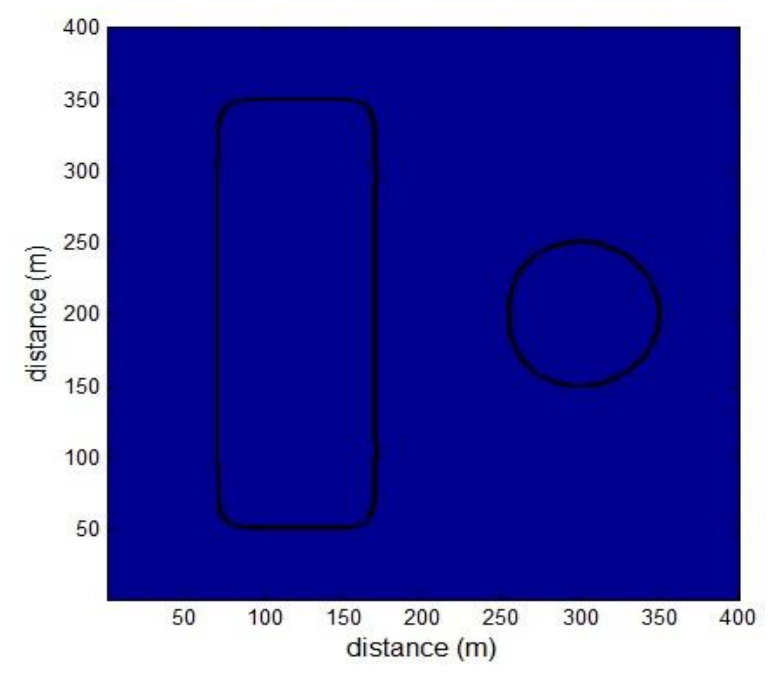

Fig. 10: The CED of the gravity data in Fig. 9. 


\section{EDGE DETECTOR CONVENTIONAL FILTERS}

There are various form of the local phase filters. The structures of these filters are based on vertical and horizontal derivatives of the potential fields. For comparison, in this paper are used of the analytic signal, tilt angle and total horizontal derivative of the tilt angle filters. These filters employ widespread for the gravity and magnetic fields interpretation as edge enhancement.

The amplitude of the analytic signal or total gradient is a commonly used filter that produces bell-shaped anomalies over magnetic bodies (Nabighian, 1972). The analytic signal is a linear combination of horizontal and vertical derivatives whose amplitude is given by:

$$
|A S|=\sqrt{\left(\frac{\partial F}{\partial x}\right)^{2}+\left(\frac{\partial F}{\partial y}\right)^{2}+\left(\frac{\partial F}{\partial z}\right)^{2}}
$$

$\mathrm{F}$ is the potential field. This filter enhances edges of features of all azimuths. Tilt angle filter is the ratio of the vertical derivative to the value of the total horizontal derivative which is defined as (Miller and Singh,1994):

$$
T=\tan ^{-1}\left(\frac{\partial F / \partial z}{\sqrt{\left((\partial F / \partial x)^{2}+(\partial F / \partial y)^{2}\right)}}\right)
$$

The zero and near to zero values of the tilt angle are located over and around the egde, where the vertical derivative is zero and the horizontal derivative is a maximum.

Verduzco et al. (2004) suggested using the total horizontal derivative of the tilt angle (which they termed the THDR) as an edge detector, i.e.

$T H D R=\sqrt{\left(\frac{\partial T}{\partial x}\right)^{2}+\left(\frac{\partial T}{\partial y}\right)^{2}}$

where $T$ is the tilt angle. The minimum values of the THDR always situate over the source while the maximum values of it pass over or near the edge.

\section{FIELD EXAMPLES}

\section{Gravity data}

The study area is located in the south-eastern Zagros fold-thrust belt and the northern part of the Persian Gulf in Hormozgan Province, Iran includes a simple anticline-syncline system (Fig. 11). Geological formation in these region is carbonates (i.e., limestone, marly limestone and calcareous marl) and gray or green marl with intercalated of thin to medium bedded limestone. Due to existence of the numerous salt domes, this area is part of Iran's petrol region. 


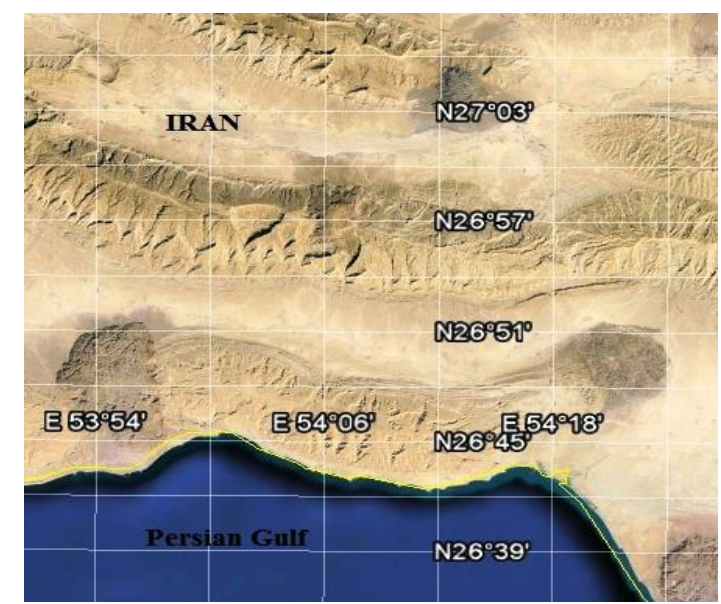

Fig.11: The satellite picture of the gravity field measurement region.

Fig. 12a shows the residual gravity anomaly map of the study region. The specified negative gravity anomaly with "SD" in the Fig. 12a is given a salt dome. The derived upper and lower threshold values of the computed gradient magnitudes from the gravity data have been presented in table 4. The threshold range is between 0.029 and 0.29 . The value of the Gaussian standard deviation $(\sigma)$ is 1 . Fig. $12 \mathrm{~b}$ shows the result of applying the CED method to the gravity data set. Fig. 12c represents the overlay of the edge map obtained by the CED algorithm (Fig. 12b) with gravity field map (Fig. 12a).

Table-4: The computed upper and lower thresholds values from the gravity field data gradient.

$\begin{array}{cccccc}\mathrm{s}_{\mathrm{F}}(\mathrm{std}) & \begin{array}{c}\mathrm{a}_{\mathrm{F}} \\ \text { (average) }\end{array} & \mathrm{m}_{\mathrm{F}}(\max ) & \min _{\mathrm{F}} & \mathrm{t}_{\text {ho }} & \mathrm{t}_{\mathrm{I}} \\ 0.0569 & 0.0959 & 0.4743 & 0.00084 & 0.29 & 0.029\end{array}$

In the overlay map, the detected borders using the CED separate the limited area of the positives and negatives gravity anomalies sources.

Because the local phase filters formula is composed of the vertical and horizontal gradients, then these filters are very susceptible to noise problems. We have been performed upward continuation of the gravity field to 0.02 degree height to weaken the short wavelengths related to the shallow sources and noise (Fig. 13).

Figs. 14, 15 and 16 show the analytic signal, tilt angle and total horizontal derivative of the tilt angle maps of the upward continued gravity field, respectively. It is expected that the maximum values of the analytic signal detect the anomalies' border, but this filter shows a week performance on the gravity data of the area under study (Fig 14).

a causative body with a positive density contrast, the tilt angle is positive when over the source and is negative outside the source region. The tilt angle could not delineate the sharp borders for the gravity anomalies in the area under consideration (Fig. 15).

Although the gravity short wavelengths related to the noise and small shallow sources in the area under study have been removed by the upward continuation method and the gravity field has been smoother, nevertheless the total horizontal derivative of the tilt angle map has been covered of noise and small anomalies (Fig. 16). The THDR has exhibited very poor results. 

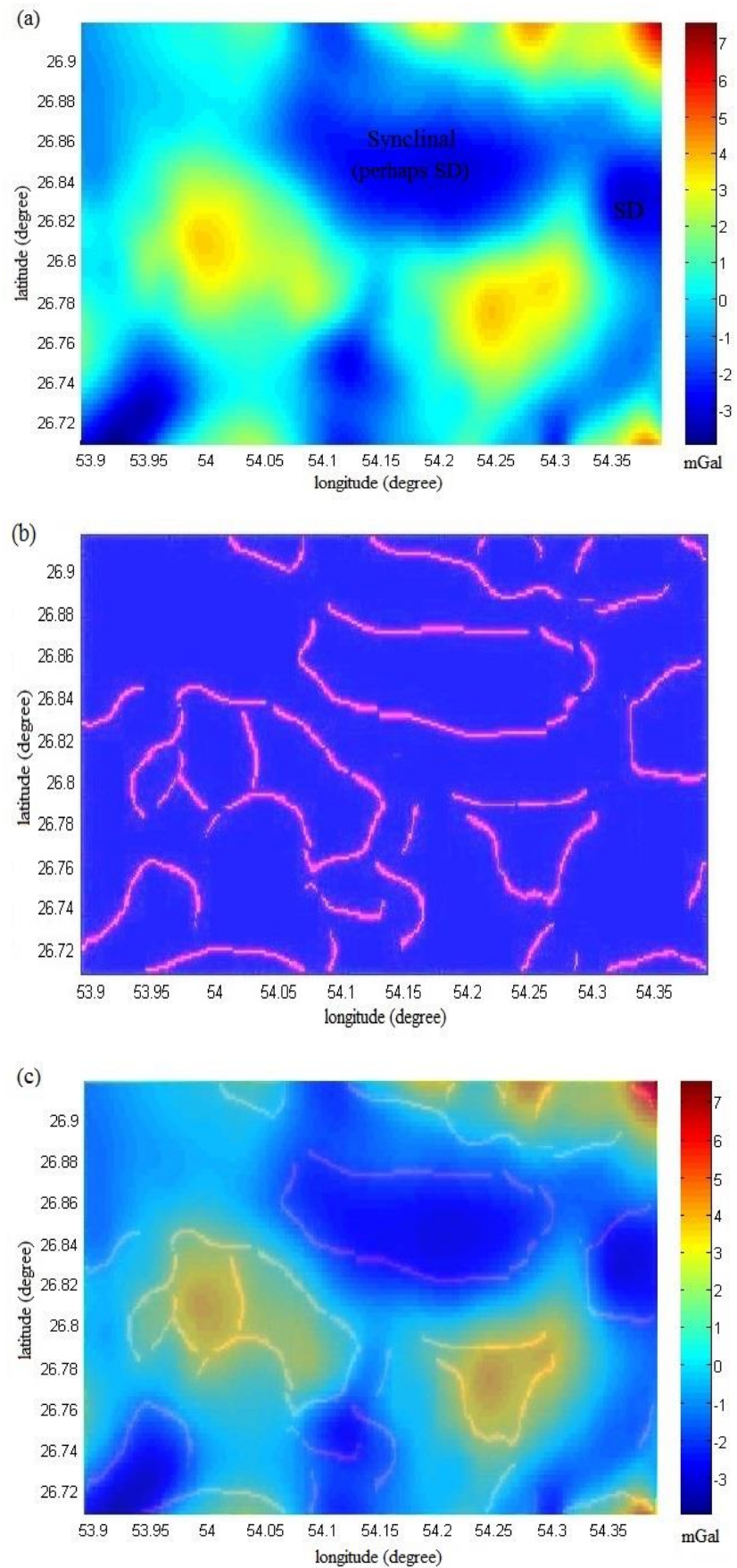

Fig.12: a) The residual gravity anomaly map of the study region in Fig. 11. b) The edge map obtained by the CED. c) The Overlay map of $a$ and $b$. 

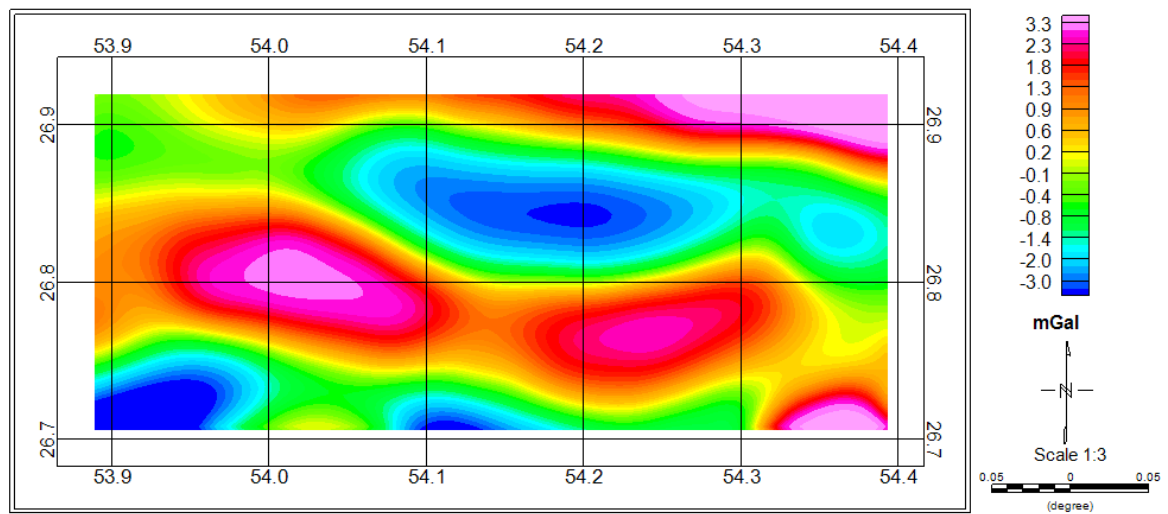

Fig. 13: The upward continuation map of the gravity field of the area under study to 0.02 degree height.
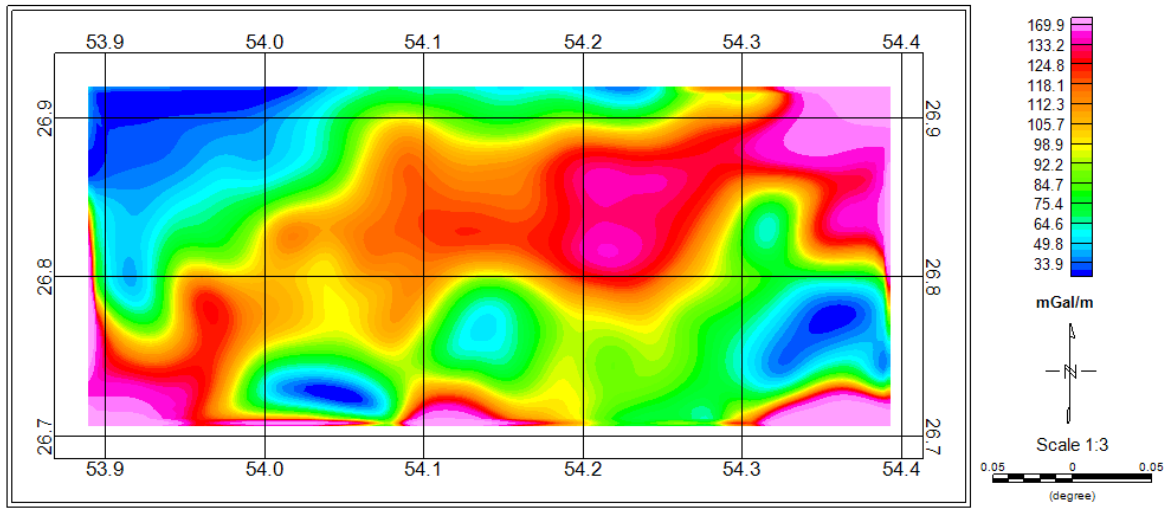

Fig. 14: The analytic signal map of the upward continued gravity field.
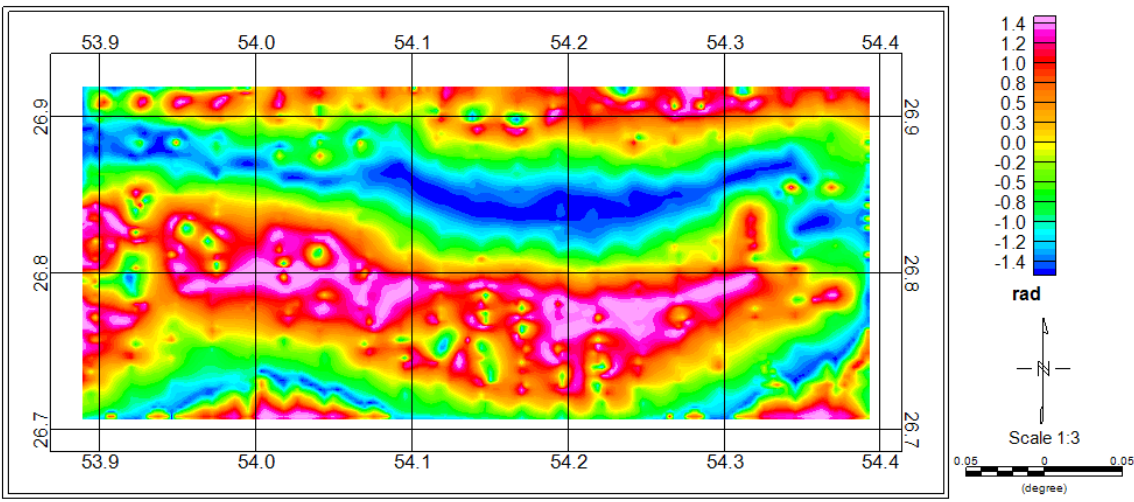

Fig. 15: The tilt angle map of the upward continued gravity field. 


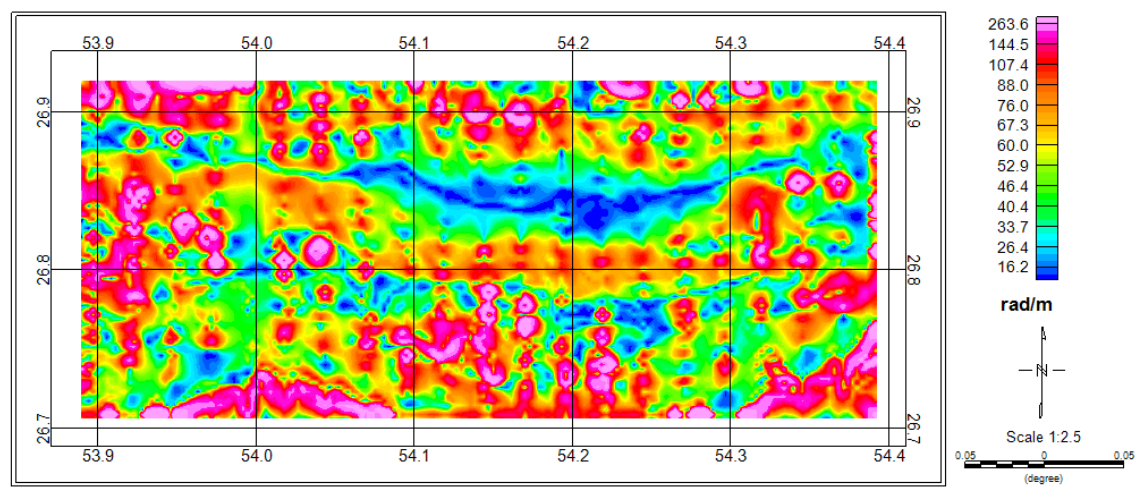

Fig. 16: The THDR of the upward continued gravity field.

\section{Magnetic data}

The under survey zone is located in Kerman province, Iran (Fig. 17). Kerman province has a long historical background in mining and geological investigation. Kerman province can be a part of Central Iran zone in structural units and extent of sedimentary basins viewpoint. Paleozoic to Mesozoic geological formation of the study region consists of dolomite and dolomitic limestone, orbitolina limestone with marl, alternations of marly biomicrite with marl, sandy micrite and siltstone. Quaternary sediments include sand dunes and sheets, silt and clay. Percambrian outcrops comprise volcanic rock, quartzite, sandstone and shale. Mineral materials such as iron, coal, copper, chromium, zinc, lead, titan and decorative building stones are abundant in this region. The main iron ores in this area are from the oxide group consisting of hematite and magnetite. The aim of the magnetic field measurments is metal resources discovery.

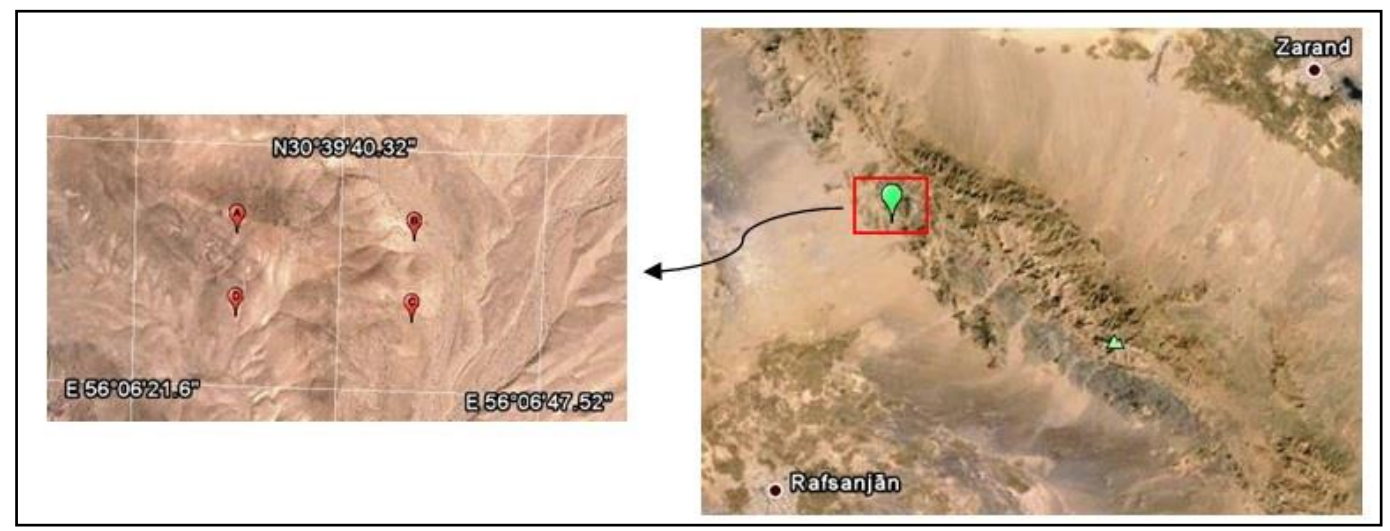

Fig. 17: The satellite picture of the study region location (right image). The red cursors in the left image show the confine of the magnetic field measurement zone.

Based on the model IGRF, the geomagnetic field inclination and declination angles in this part are $46.99^{\circ}$ and $2.8^{\circ}$, respectively. The magnetic reduction to pole data is shown in Fig. $18 \mathrm{a}$ while for elimination the short wavelength from shallow sources has been used the upward continuation to height of $10 \mathrm{~m}$ and geological structure or metal deposits with high magnetic susceptibility are located in the relatively high magnetic anomaly area. Table-5 indicates the upper and lower threshold values that are deduced of the gradient magnitudes of the magnetic field. 
The upper and lower threshold values are 0.24 and 0.044 . The value of the Gaussian standard deviation $(\sigma)$ is 1 . Fig. 18b shows the edge map of the magnetic field data in the Fig. 18a.
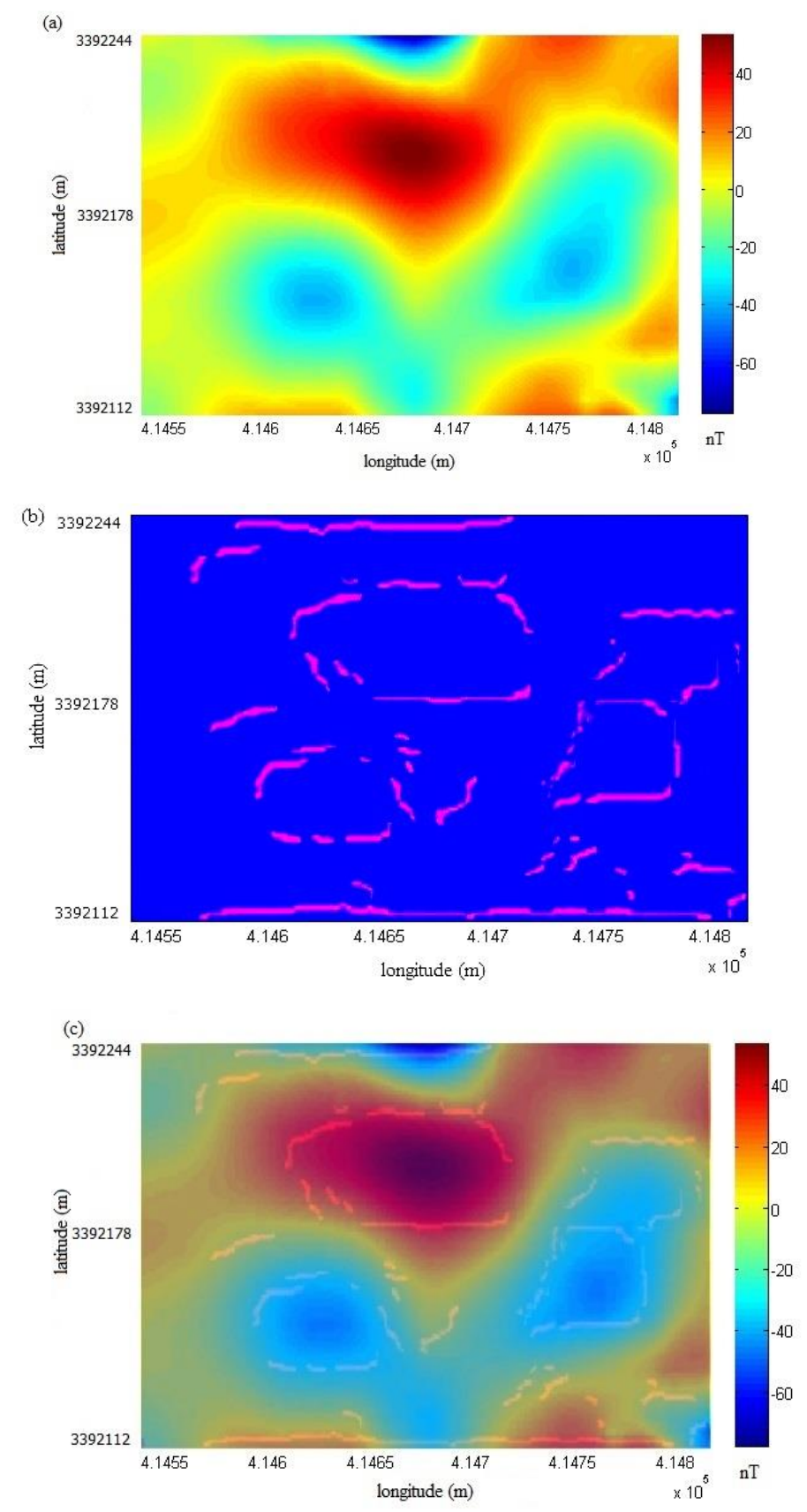

Fig.18: a) Reduction to pole magnetic anomaly with upward continuation $10 \mathrm{~m}$ height. b) the edge map obtained by the CED. c) The overlay map of $a$ and $b$. 
Table-5: The computed upper and lower thresholds values obtained from the magnetic field data gradient.

$\begin{array}{cccccc}\mathrm{s}_{\mathrm{F}}(\mathrm{std}) & \begin{array}{c}\mathrm{a}_{\mathrm{F}} \\ \text { (average) }\end{array} & \mathrm{m}_{\mathrm{F}}(\max ) & \min _{\mathrm{F}} & \mathrm{t}_{\text {ho }} & \mathrm{t}_{\text {I }} \\ 0.818 & 1.154 & 5.938 & 0.0019 & 0.24 & 0.044\end{array}$

The overlay map (Fig. 18c) displays the location of the underground magnetic anomalies outskirt. The limitation of the magnetic anomalies by analytic signal map (Fig.19) is almost impossible. The border of the magnetic anomaly sources have been somewhat revealed by zero values of the tilt angle, but determine a ceaseless and hairline border is difficult and stickler (Fig. 20). As can be seen from Fig. 21, the interpreting the total horizontal derivative of the tilt angle map for determining the boundary location is very intricate.
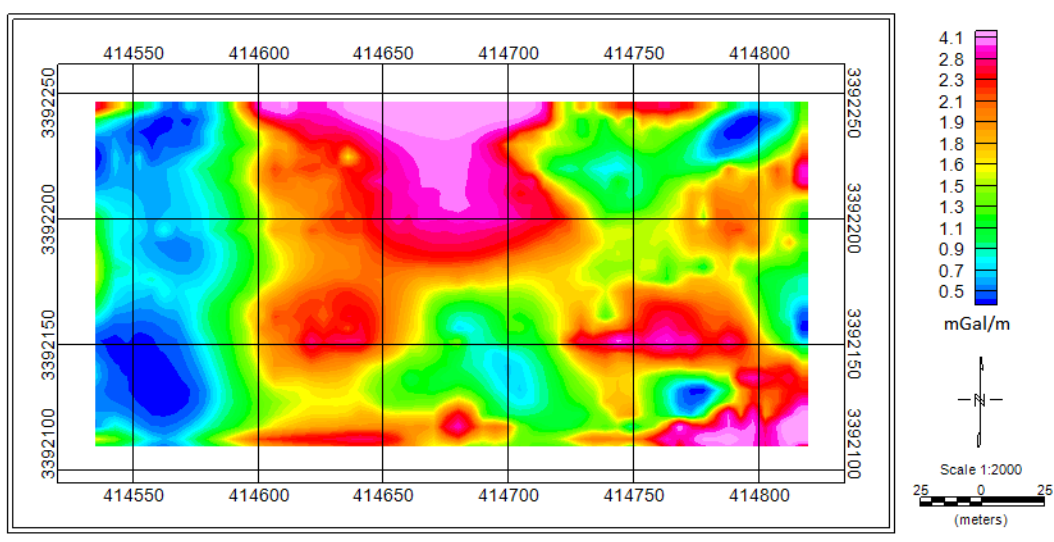

Fig. 19: The analytic signal map of the magnetic field.
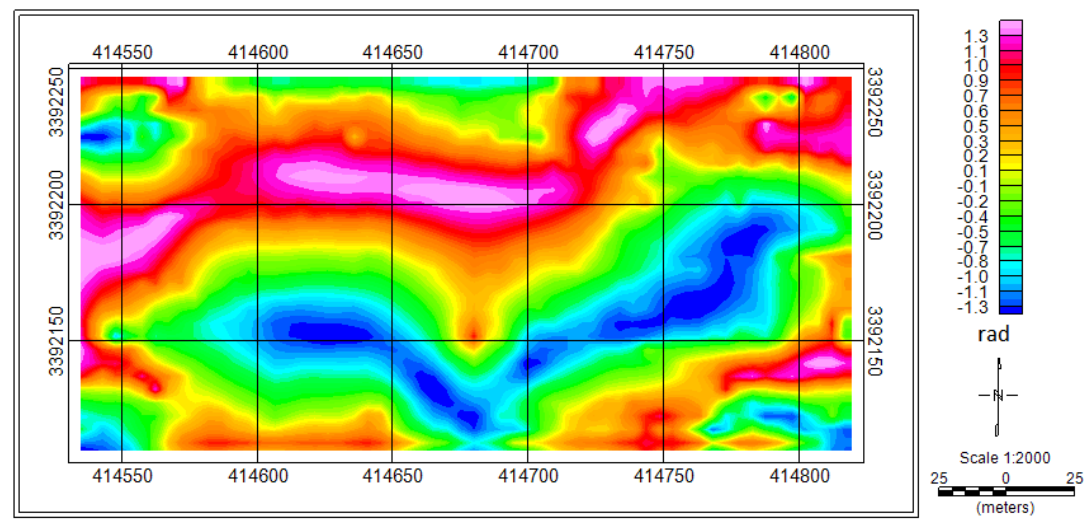

Fig. 20: The tilt angle map of the magnetic field. 

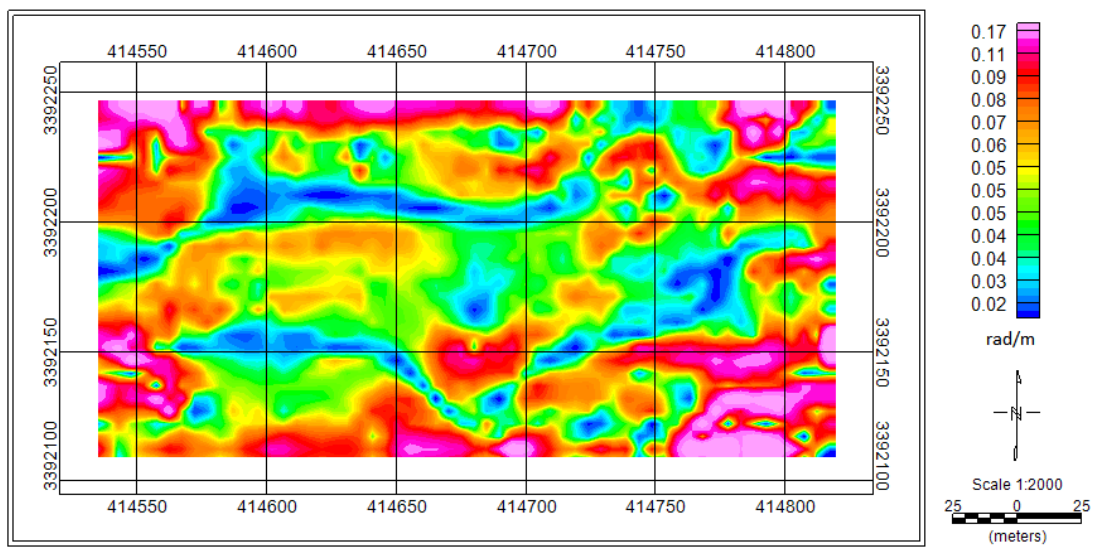

Fig. 19: The THDR map of the magnetic field.

\section{DISCUSSION AND RESULTS}

In this paper, we are demonstrated the efficiency of the canny method as a edge detector for the potential maps. The effect of the canny operator is estimated by three parameters; the width of the Gaussian kernel used in the smoothing phase, and the upper and lower thresholds used in the enhancement phase. The accurate performance of the CED method in the synthetic gravity and magnetic field processing is representative of the recommended procedures efficiency for determining the thresholds values. This study exemplify that the CED analysis can be a very powerful tool in detecting and mapping lineaments in the potential field maps. The narrow and sharp borders resulted of the CED separate the causative body edges of the positives and negatives magnetic and gravity anomalies. The edge thickness in the canny method depends on the pixel size. The tenuous exhibition of the anomaly source' border is one of the great advantages of the CED compared to common methods such as analytical signal, tilt angle and total horizontal derivative of the tilt angle. The boundary determination results confirm that the CED is an effective and flexible technique for the potential fields analysis, particular when the signal to noise ratio be low. Whiles the forgoing edge detector conventional filters were not able to illustrate a distinct and clear boundary, the CED represents the acceptable results.

\section{REFERENCES}

Boschetti, F. (2005) Improved edge detection and noise removal in gravity maps via the use of gravity gradients. J. Appl. Geophys., v. 57, pp. 213-225.

Canny J.F. (1986) A computational approach to edge detection. IEEE Trans Pattern Analysis and Machine Intelligence, v. 8(6), pp.679-698.

Cooper, G.R.J. and Cowan, D.R. (2008) Edge enhancement of potential-field data using normalized statistics. Geophysics, v. 73 (3), p. h1-h4. 10.1190/1.2837309.

Davis, L. S. (1995) Edge detection techniques. Computer Graphics Image Process., v. 4, pp. 248-270.

Eshaghzadeh, A. (2014) Anomaly edge enhancement of microgravity data using normalized standard deviation. Geodynamics Research International Bulletin, v. 2(3), pp. XLVIII to LII.

Eshaghzadeh, A. (2015) Image edge detection of the total horizontal gradient of gravity data using the normalized tilt angle. Geodynamics Research International Bulletin, v. 3(4), pp. XXVIII to XXXIII. 
Eshaghzadeh, A. (2016) gravity anomalies edge intensification using the tilt angle of the balanced total horizontal derivative. Geoinformatics: Theoretical and Applied Aspects., 10-13 May, Kiev, Ukraine.

Lili, Li., Guoqing, Ma. and Xiaojuan, Du. (2013) Edge detection in potential-field data by enhanced mathematical morphology filter. Pure and Applied Geophysics., v. 170(4), pp 645-653.

Miller, H.G. and Singh, V. (1994) Potential field tilt- a new concept for location of potential field sources. Journal of Applied Geophysics., v. 32, pp. 213-217.

Nabighian, M. N. (1972) The analytic signal of two dimensional magnetic bodies with polygonal cross section: Its properties and use for automated anomaly interpretation. Geophysics, v. 37, pp. 507517.

Narayan, Satya Sahoo, Soumyashree Debasis, Pal, S. K., Kumar, Ujjawal, Pathak, Vipin Kumar, Majumdar, T. J. and Chouhan, Avinash (2016) Delineation of structural features over a part of the Bay of Bengal using total and balanced horizontal derivative techniques. Geocarto. International., v. 32(1), pp. 116.

Pala, S.K. and Majumdarb, T.J. (2015) Geological appraisal over the Singhbhum-Orissa Craton, India using GOCE, EIGEN6-C2 and in-situ gravity data. International Journal of Applied Earth Observations and Geoinformation., v. 35, pp. 96-119.

Pilkington, M. and Keating, P. (2004) Contact mapping from gridded magnetic data- a comparison. Extended abstracts, ASEG 17th Geophysical Conference and Exhibition, Sydney.

Rao, D. B. and Babu, N. R. (1991) A rapid method for three-dimensional modeling of magnetic anomalies. Geophysics., v. 56(2), 1729-1737.

Thurston, J.B. and Smith, R.S. (1997) Automatic conversion of magnetic data to depth, dip, and susceptibility contrast using the SPI (TM) method. Geophysics., v. 62, pp. 807-813.

Verduzco, B., Fairhead, J.D. and Green, C.M. (2004) New insights into magnetic derivatives for structural mapping. The Leading Edge., v. 23 (2), pp. 116-119.

Wijns, C., Perez, C. and Kowalczyk, P. (2005) Theta map: edge detection in magnetic data. Geophysics., v. 70 (4), pp. 39-43.

(Received: 11.09.2016; Accepted: 28.06.2017) 